\title{
The Magnetic Disaccommodation in Titanium Doped Magnetite
}

\author{
K. Bendimya, C. de Francisco, P. Hernandez, O. Alejos and J.M. Muñoz \\ Dpto. Electricidad y Electronica, Facultad de Ciencias, Universidad de Valladolid, \\ 47071 Valladolid, Spain
}

\begin{abstract}
The domain wall stabilization processes in titanium doped magnetite is investigated by means of disaccommodation techniques. The time evolution of the initial magnetic susceptibility after sample demagnetization is measured in temperature range 80 to $500 \mathrm{~K}$ for polycrystalline specimens with nominal composition, $\mathrm{Fe}_{3 \times \mathrm{x}} \mathrm{Ti}_{\mathrm{x}} \mathrm{O}_{4},(0<\mathrm{x}<0.1)$. The presence of $\mathrm{Ti}^{4+}$ ions in the spinel lattice changes drastically the disaccommodation spectra observed in vacancy-doped magnetite. The increase of the tetravalent cations in the lattice involves an almost linear decrease of the Verwey temperature and the splitting of processes III $(300 \mathrm{~K})$ into $\left(\mathrm{IV}^{\prime}, \mathrm{IV}, \mathrm{III}\right)$ at temperatures close to $215 \mathrm{~K}, 260 \mathrm{~K}$ and $300 \mathrm{~K}$ respectively. Furthermore, a new long-range diffusion contribution appears in temperature region II, whereas peak $I$ increases its relative contribution. The origin of the observed phenomena is discussed in terms of $\mathrm{Fe}^{2+}$ ions jumps into the vacancies as in $\mathrm{Fe}_{3} \mathrm{O}_{4}$ (process $\mathrm{m}$ ), jumps of $\mathrm{Fe}^{2+}$ into a vacancies in octants with a $\mathrm{Ti}^{4+}$ (process $\mathrm{IV}$ ) and jumps of $\mathrm{Fe}^{2+}$ which lies on octants with a $\mathrm{Ti}^{4+}$ into vacancies (process $\mathrm{IV}^{\prime}$ ).
\end{abstract}

\section{INTRODUCTION}

In this work we present a preliminary study dealing with magnetic aftereffect in ceramic titanium ferrites. We begin by analyzing the relaxation of the domain wall mobility in samples with low Ti contents. This phenomenon is investigated by means of a magnetic disaccommodation technique, in which the time evolution of the reversible magnetic permeability is measured after sample demagnetization. A comparison of relaxation spectra with those obtained for magnetite samples, reveals that low Ti substitution levels affect drastically the observed relaxation. Finally, the mechanisms responsible for this behavior are discussed.

\section{EXPERIMENTAL PROCEDURE}

\subsection{Sample preparation}

A series of polycrystalline samples with nominal composition $\mathrm{Ti}_{\mathrm{x}} \mathrm{Fe}_{3-\mathrm{x}} \mathrm{O}_{4}(0 \leq \mathrm{x} \leq 0.1)$ has been prepared from stoichiometrically mixed high purity $\mathrm{Fe}_{2} \mathrm{O}_{3}$ and $\mathrm{TiO}_{2}$ powders. The mixture were ground, pressed in a cylindrical die and fired for 8 hours at $1400{ }^{\circ} \mathrm{C}$ in a $\mathrm{CO}_{2}\left(\mathrm{P}_{\mathrm{O}_{2}} \approx 10^{-3} \mathrm{~atm}\right)$ atmosphere according to Ti-Fe-O phase diagrams [1]. Finally, rapid temperature quenching was performed to avoid vacancy.annealing. Under constant oxygen pressure, vacancy concentration increases with $\mathrm{Ti}$ content [1].

\subsection{Measurement technique}

Magnetic disaccommodation measurements have been carried out with the help of a computer-aided system based of an automatic LCR bridge[2]. In the measuring process, the relative variation of the initial permeability after sample demagnetization is recorded at different rate windows with scanning temperature ( 80 to $500 \mathrm{~K})$. The experimental results are then processed by using the analysis techniques described in [3]. The samples were demagnetized by means of a linearly decaying ac field, whose amplitude and signal duration have been checked in order to obtain reproducible results.

\section{EXPERIMENTAL RESULTS}

\subsection{Relaxation spectra}

Figure 1 displays the isochronal spectra for different $x$-values. We can observe that in the scanned temperature range five clear peaks emerge at temperatures close to $215,260,300,400$ and $445 \mathrm{~K}$ (hereafter as IV', IV, III, II and I-peak). These 
processes exhibit the characteristics of thermally activated relaxations, and their features are strongly modified by the Ti content. Also we can note that the increase of the tetravalent cations involves the splitting of process III into three peaks (IV', IV, III). Peak III shows a progressive decrease of his relative amplitude, whilst the peak IV' becomes preponderant for high Ti content. Furthermore, the temperature position of all peaks shifts to low temperatures and a new long-range diffusion contribution appears in the temperature region II. Finally the peak I increases its relative contribution and its width. In addition, in low temperature region we can observe an almost linear decrease of the Verwey temperature (see figure 2).

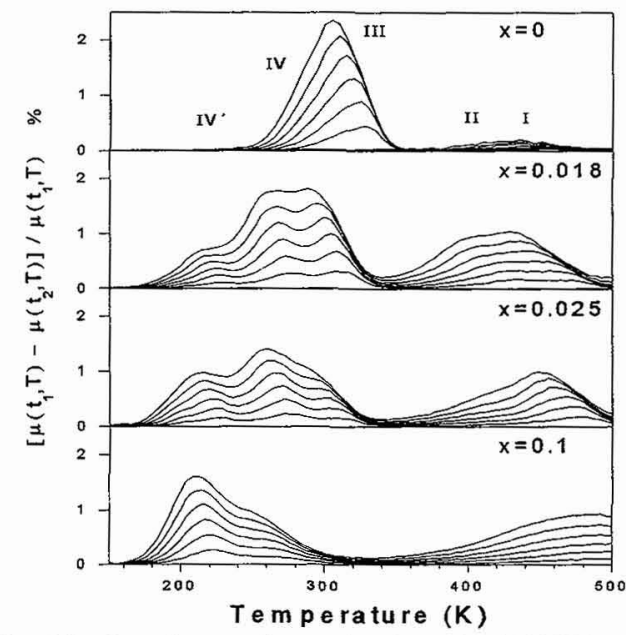

Fig. 1. Isochronal curves for samples sintered at $1400^{\circ} \mathrm{C}$ in $\mathrm{CO}_{2}$ atmosphere. $\left(t_{1}=2 \mathrm{~s}\right.$ and $t_{2}=4,8,16,32,64,128 \mathrm{~s}$, curves from bottom to top )

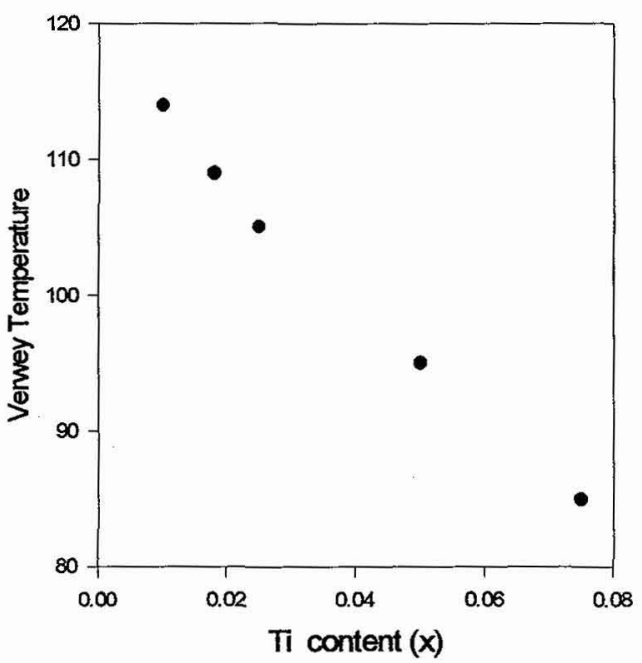

Fig. 2. Verwey temperature of the spinels $\mathrm{Ti}_{\mathbf{x}} \mathrm{Fe}_{3-x} \mathrm{O}_{4}$ for different titanium contents.

\subsection{Numerical fitting}

The numerical analysis of the isochronal curves reveals that processes IV', IV, III and II can be successfully fitted by means of the superposition of Debye processes. Its activation energies are close to $0.56-0.6 \mathrm{eV}, 0.67-0.73 \mathrm{eV}, 0.78-0.82 \mathrm{eV}$ and $1.05-$ $1.15 \mathrm{eV}$ respectively, and preexponential factor equal to $10^{-13} \mathrm{~s}$. On the other hand, the peak I seems to be related to a broad distribution of activation energies centered around $1.25 \mathrm{eV}$.

\section{DISCUSSION}

For $\mathrm{Fe}_{3} \mathrm{O}_{4}$, it is suggested that the magnetic disaccommodation is caused by the formation of an induced anisotropy due to jumps of octahedral $\mathrm{Fe}^{2+}$ into vacancies. Two processes are present, depending whether the B-site neighbors of jumping $\mathrm{Fe}$ ion are $\mathrm{Fe}^{3+}$ or $\mathrm{Fe}^{2+}$, two different octants can be distinguished. Thus, the relaxation process take place with two activation energies close to 0.82 and $0.88 \mathrm{eV}$ [4]. The same phenomenon may occur in $\mathrm{Ti}_{\mathrm{x}} \mathrm{Fe}_{3-\mathrm{x}} \mathrm{O}_{4}$. When the Ti content increases, the number of the octants with the quasi-free electron also increases and the process III splits into three peaks: the process III as in pure magnetite $\left(\mathrm{Fe}_{3} \mathrm{O}_{4}\right)$ and the processes IV and IV' at lower temperature. These last processes are due to $\mathrm{Fe}^{2+}$ jumps into vacancies in the neighborhood of $\mathrm{Ti}^{4+}$, which produce a local distortion of the crystal structure and so decreases the activation energies, appearing two peaks. Discussed in terms of $\mathrm{Fe}^{2+}$ jumps into vacancies in octants with a $\mathrm{Ti}^{4+}$ (process IV) and jumps of $\mathrm{Fe}^{2+}$ ions which lies on octants with a $\mathrm{Ti}^{4+}$ ion into a vacancy (process IV'). Taking into account their origin, each individual peak is fitted by two Debye terms. In addition, the large increase in the disaccommodation amplitude at high temperature is associated with $\mathrm{Ti}^{4+}$ jumps and the ordering of ferrous-ion pairs through the agency of the diffusion of the vacancies, which do not contribute themselves to the induced anisotropy (processes II and I). In the range of low temperatures, the distortion induced by the $\mathrm{Ti}^{4+}$ ions involves a increases of the lattice parameter and a shifting to lower temperature of the Verwey temperature transition (see figure 2).

This work has been partially supported by Junta de Castilla y Leon (VA25/94)

\section{References}

[1] Aragon R. and McCallister R.H., Phys Chem Minerals 8 (1982) 112-120.

[2] De Francisco C., Muñoz J.M., and Ayala J., IEEE Trans. on mag. MAG-23(2) (1987) 1866-1868.

[3] De Francisco C., Iñiguez J. and Muñoz J.M., IEEE Trans. on Mag. MAG-25(6) (1989) 4413-4420.

[4] Kronmüller H. and Walz F., Philosophical Magazine B 42 (1980) 433-452. 